\title{
FREQUENCY response of underwater ultrasonic transducers in the near field using polarimetric polarization maintaining fiber sensors
}

\author{
Abdeldjalil Bennecer ${ }^{* a}$, Michelle McGuire, ${ }^{\mathrm{a}}$, Gordon M. H. Flockhart ${ }^{\mathrm{a}}$, S. Gareth Pierce ${ }^{\mathrm{a}}$, Gordon \\ Hayward $^{\mathrm{a}}$, Brian Culshaw ${ }^{\mathrm{a}}$ \\ ${ }^{a}$ Dept. of Electrical and Electronic Eng., Univ. of Strathclyde, 204 George St., Glasgow, G1 1XW, \\ $\mathrm{UK}$;
}

\begin{abstract}
Near-field ultrasonic response $(50-2000 \mathrm{kHz})$ of an underwater 1-3 piezocomposite transducer is experimentally investigated using a polarimetric polarization maintaining fiber sensor. Measured outputs from our sensor and a reference hydrophone are observed to be comparable.
\end{abstract}

Keywords: Fiber optic sensor, polarimetry, ultrasonic sensor

\section{INTRODUCTION}

Ultrasonic optical fiber sensors (UOFSs) offer many advantages over other sensing methodologies. These include greater sensitivity, smaller size, reduced weight and low cost as well as being reliable and versatile. In addition, their immunity to electromagnetic interference and their compatibility to optical communication make them strong candidates of choice. Since the invention of optical fibers, they have been actively investigated for a range of ultrasound detection applications such as structural health monitoring ${ }^{1}$, imaging ${ }^{2}$ and sonar ${ }^{3}$. As a result, a number of UOFS techniques based mainly on interferometry, polarimetry or fiber Bragg grating $(\mathrm{FBG})^{4}$, have been developed. Each of these techniques has strengths as well as weaknesses depending on the target application.

We are interested in directly monitoring the acoustic output of ultrasonic arrays or sonar systems. Often, Polyvinylidene Fluoride (PVDF) film is used but this needs to be located on the transducer surface to be able to accurately monitor its output. Unfortunately, such PVDF films are prone to electromagnetic interference and thus impractical for our purpose. Here, we propose the use of polarimetric polarization maintaining fiber (PMF) sensor instead. The validity of a polarimetric sensor as a monitor of high frequency acoustic has been demonstrated by Flax et al. ${ }^{5}$ while the sensitivity of a PMF has also been shown to be higher than that of a single mode fiber (SMF) ${ }^{6}$. In this paper, we report the investigation of a PMF sensor that is capable of directly measuring the acoustic frequency response $(50-2000 \mathrm{kHz})$ of an underwater ultrasonic transducer.

\section{EXPERIMENT}

The experimental arrangement for an underwater ultrasonic polarization maintaining fiber (PMF) sensor based on the principle of polarimetric interference is depicted in Figure 1. Light from a narrow-band tunable laser source (Santec TSL320) becomes linearly polarized after it travels through an in-line polarizer. The laser is operated in constant power (5.4 $\mathrm{mW})$ mode and with a fixed wavelength $(1550 \mathrm{~nm})$ throughout the measurements. The polarization controller (PC) is used to ensure maximum transmission intensity. A piece of a bow-tie PMF from Fibercore with a beat length of $3.67 \mathrm{~mm}$ at the operating wavelength and which effectively constitutes the sensing region, is spliced at $45^{\circ}$ so that light power is split equally in both its axes. Then, a short length of this sensing fiber is supported by a rotatable fiber holder, where a bare PMF $(125 \mu \mathrm{m}$ of diameter) is made to pass across the front face of an ultrasonic transducer in order to monitor its output in the near-field region. The ultrasonic transducer (Alba AD-017-002) of $58 \mathrm{~mm}$ diameter is a 1-3 piezocomposite

* abdeldjalil.bennecer@eee.strath.ac.uk; phone +44 141 548-3411; fax +44 141 548-2926; www.strath.ac.uk/eee/

Fourth European Workshop on Optical Fibre Sensors, edited by José Luís Santos, Brian Culshaw, José Miguel López-Higuera, William N. MacPherson, Proc. of SPIE Vol. 7653, 76530S

(C) 2010 SPIE · CCC code: $0277-786 \mathrm{X} / 10 / \$ 18 \cdot$ doi: $10.1117 / 12.866863$

Proc. of SPIE Vol. 7653 76530S-1 
transducer with its first resonant frequency at $250 \mathrm{kHz}$ in water. The transducer and the fiber sensor are both submerged in water.

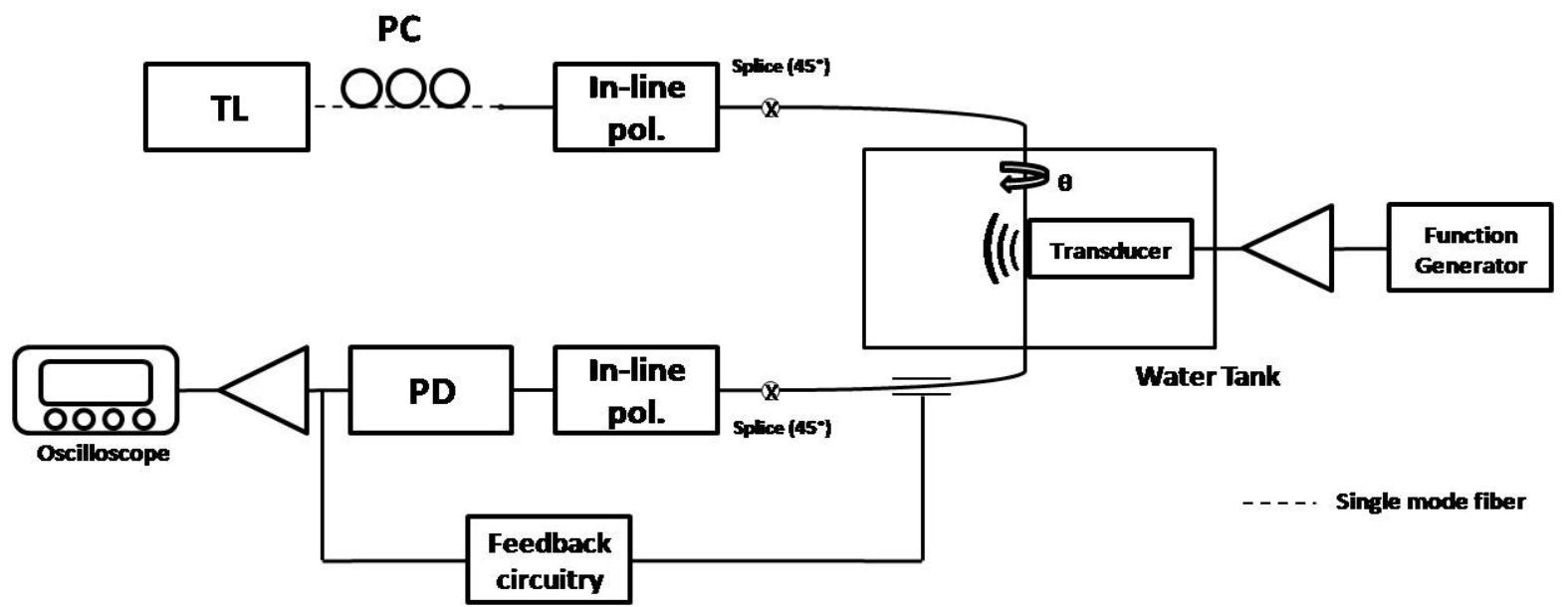

Figure 1. Experimental set-up of a polarimetric PMF sensor.

The cross section of a bare bow-tie PMF is shown in Figure 2, where the slow and fast axes of the fiber denoted by the $\mathrm{x}$ and y axes, respectively, and an ultrasonic wave is assumed to be incident normally in the direction of the fiber at an angle $\theta$ to the $\mathrm{x}$ axis. The propagation direction of the ultrasound and its normal on the $\mathrm{xy}$ plane are denoted by the $\mathrm{x}^{\prime}$ and $y^{\prime}$ axes, respectively. The radial compression resulting from the ultrasonic field is therefore translated to a modulation of the phase difference between the two orthogonal axes. At the detection side, another $45^{\circ}$ splice is made before light is launched into another in-line polarizer. The interference of the two waves produces an interference waveform which is detected by a photodetector and amplifier (Femto,OE-200-IN). The signal is displayed on an oscilloscope. Finally, in order to obtain optimum sensitivity, the light is set at the quadrature point (i.e. circularly polarized) at the second $45^{\circ}$ splice. This is achieved through a feedback electronic controller circuit and a thermo-electric module.

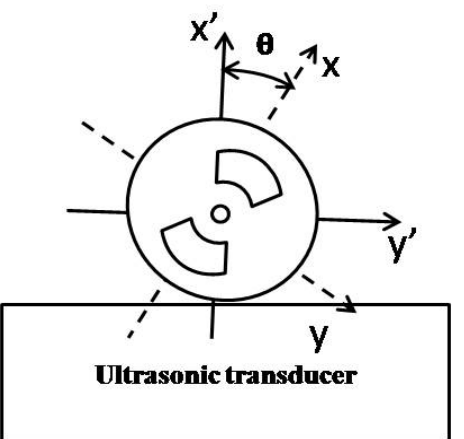

Figure 2. Cross section view of a bare bow-tie PMF sensor in the near field region of an ultrasonic transducer

\section{RESULTS AND DISCUSSION}

\subsection{Narrowband excitation}

An arbitrary waveform generator (Agilent 33250A) is used to provide a narrow band excitation (10-cycle Hamming windowed tonebursts at 250, 909 and $1550 \mathrm{kHz}$ ) to an RF power amplifier (ENI 2100L) to drive the transducer with a toneburst of $80 \mathrm{~V}$ peak-to-peak. The response of the sensor is captured at the oscilloscope and the waveform is shifted in time to align with the highest peak of the transducer drive signal. The comparison between the two signals as shown in Figure 3 indicates a high degree of correlation. 


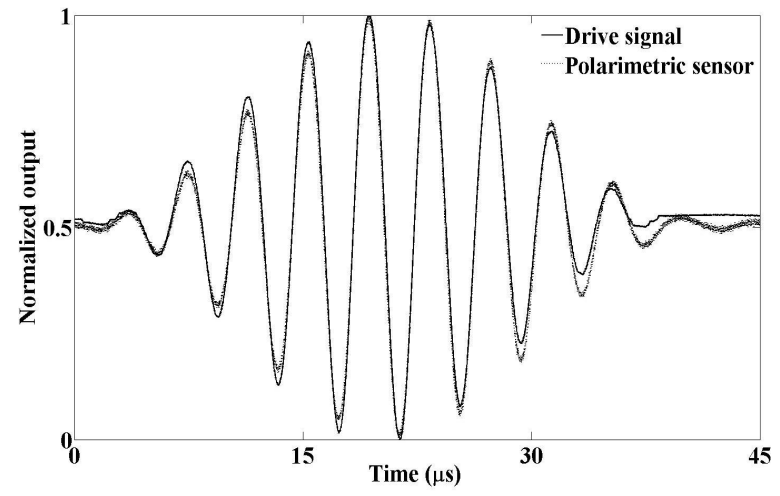

Figure 3. Time domain comparison of a polarimetric PMF sensor output and a transducer drive signal under 10 cycle toneburst excitation at $250 \mathrm{kHz}$ and $80 \mathrm{~V}$ peak-to-peak $\left(\theta=90^{\circ}\right)$.

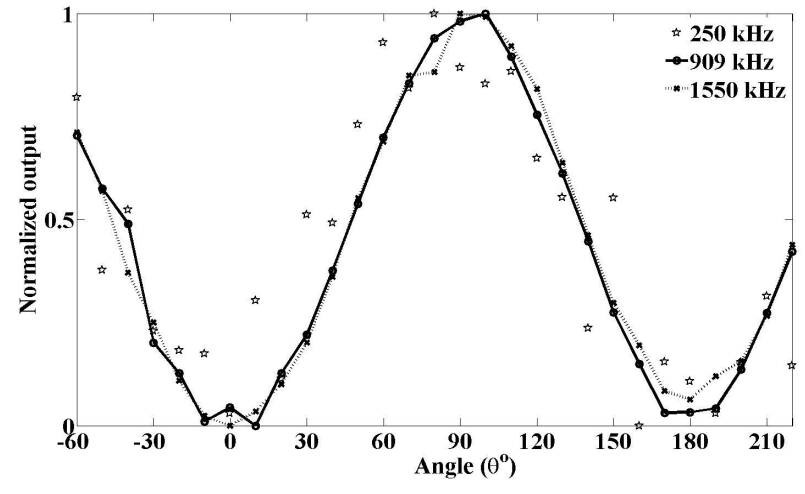

Figure 4. Normalized sensor output at the ultrasonic frequencies of 250,909 and $1550 \mathrm{kHz}$ as a function of the fiber orientation.

These measurements are then repeated for different fiber orientations in order to determine the fiber axes alignment that provides maximum sensitivity. The measured and normalized peak-to-peak values from the sensor output are plotted in Figure 4 as a function of the fiber angle for ultrasonic frequencies of 250,909 and $1550 \mathrm{kHz}$. These results show that at such ultrasonic frequencies, the ultrasonic pressure acting on the fiber is considered to be uniform and isotropic ${ }^{6}$. Therefore, the intrinsic birefringence induced by the transducer output is more dominant in this case. It is also noteworthy that there are no variations in the waveform as the orientation of the fiber is modified. Another property of interest is the angle dependence on the ultrasonic frequency. This can be shown in Figure 4 without normalizing the sensor voltage peak-to-peak. Here, instead, the visibility ( = amplitude/average ) of the measured waveform fringes as a function of fiber orientation is calculated and summarized in Table 1.

Table 1. Calculated visibility of angle dependence as a function of ultrasonic frequency.

\begin{tabular}{|l|l|}
\hline \multicolumn{1}{|c|}{ Ultrasonic frequency (kHz) } & \multicolumn{1}{c|}{ Visibility } \\
\hline 250 & 0.0216 \\
\hline 909 & 0.1066 \\
\hline 1550 & 0.6884 \\
\hline
\end{tabular}

\subsection{Broadband excitation}

A wideband excitation is applied to the transducer and the polarimetric fiber sensor response is recorded for different fiber orientations. The excitation source is a single $600 \mathrm{~ns}$ pulse with an amplitude of $70 \mathrm{~V}$, sufficiently wideband $(\sim 2$ $\mathrm{MHz})$ to produce an impulse response in the transducer. In Figure 5, the time and frequency domain response of the ultrasonic transducer where the PMF sensor show the highest and lowest sensitivities, are plotted. It is worth noting that for ultrasonic frequencies $\geq 2 \mathrm{MHz}$, the induced birefringence on the PMF sensor output starts to show more of the anisotropic regime.

From these measurements, the maximum sensitivity is determined to be at an angle $\theta=90^{\circ}$ where the fast axis of the PMF coincides with the ultrasound direction as discussed in $^{6}$. The polarization maintaining fiber is then embedded at that angle in a $250 \mu \mathrm{m}$ layer of polyurethane, which is cast onto the transducer surface. The polyurethane layer is selected to have acoustic impedance similar to water to minimize acoustic reflections within the layer. The layer of polyurethane has a longitudinal velocity of $1542 \mathrm{~m} / \mathrm{s}$, a density of $1081 \mathrm{~kg} / \mathrm{m}^{3}$ and an acoustic impedance of around 1660 MRayl. An impulse is applied to the transducer and ultrasounds are measured using the PMF sensor. A polyvinylidene fluoride (PVDF) membrane hydrophone (GEC Marconi Y-34-3598) calibrated at around $900 \mathrm{kHz}$ with an amplifier (Cooknell Electronics Ltd. CA7/C) is also used to monitor the acoustic output at some distance. The two outputs agree well as shown in Figure 6. 

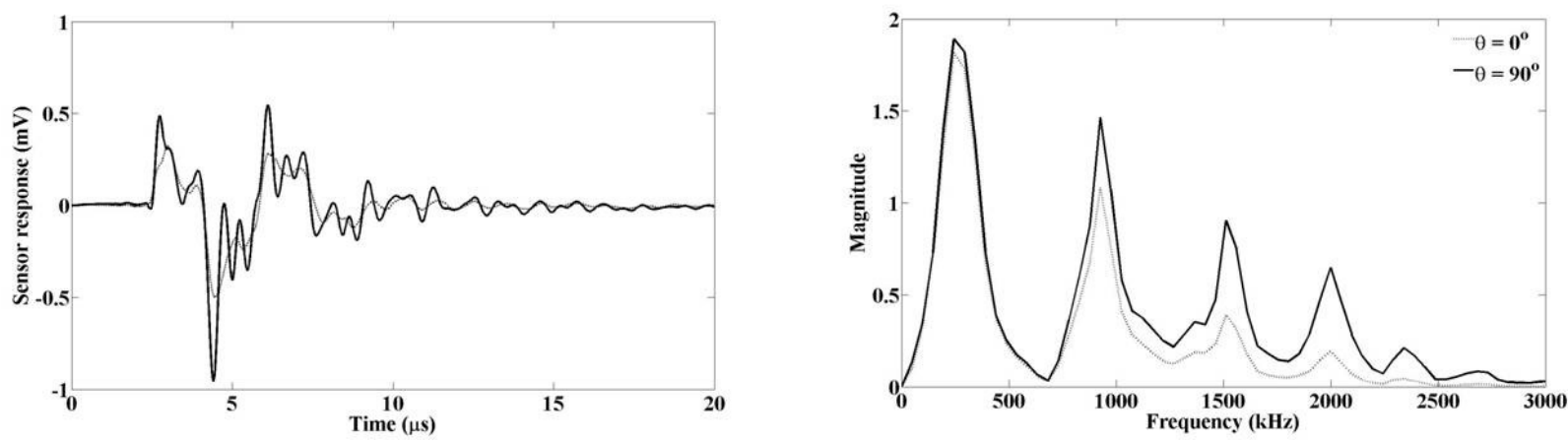

Figure 5. Time (left) and frequency (right) response of an ultrasonic transducer using a PMF sensor for $\theta=0^{\circ}$ and $90^{\circ}$.
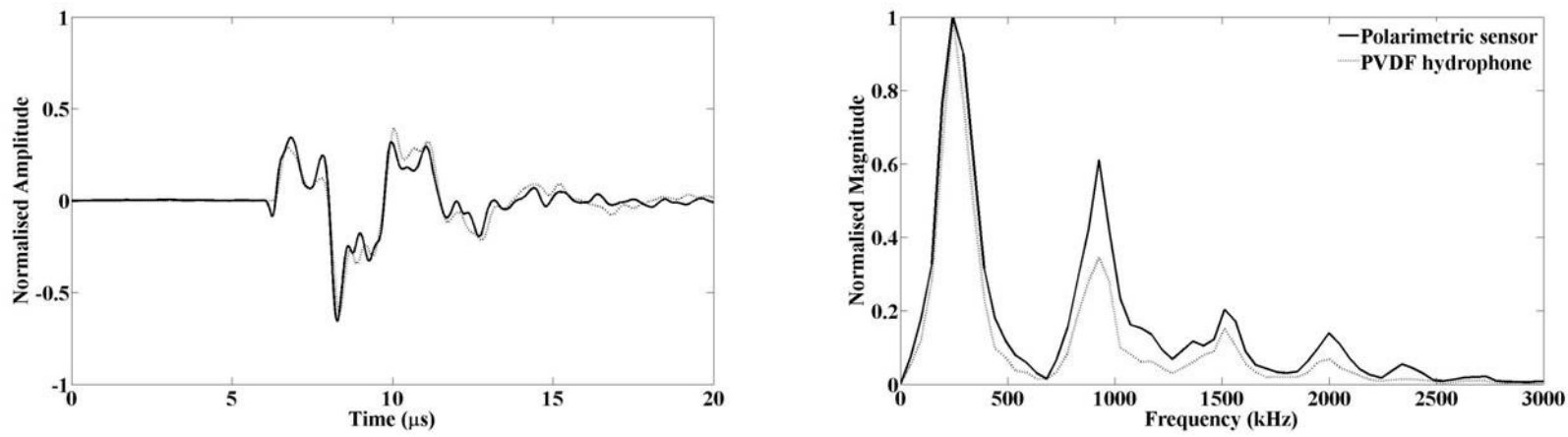

Figure 6. Comparison of a polarimetric PMF sensor (embedded in $250 \mu \mathrm{m}$ layer of polyurethane) output and a PVDF hydrophone output in time (left) and frequency (right) domain.

\section{CONCLUSION}

We demonstrated preliminary results of a polarimetric PMF sensor that is sensitive to the acoustic output of an underwater 1-3 piezocomposite transducer in the near field region. The sensitivity is shown to be dependent on the fiber orientation, albeit this dependency starts to diminish at very low ultrasonic frequencies (less than $250 \mathrm{kHz}$ ). The transducer frequency response recorded from a PVDF hydrophone agreed well with the one obtained from our fiber sensor. Nevertheless, further work remains to determine minimum detectable acoustic pressure in order to fully exploit the PMF sensor capabilities to monitor transducers output in the near field region.

\section{REFERENCES}

[1] Thursby, G., Sorazu, B., Betz, D., Staszewski, M. and Culshaw, B., "The use of fibre optic sensors for damage detection and location in structural materials," J. App. Mech. Mat. 1-2, 191-196 (2004).

[2] Fisher, N. E., Surowiec, J., Webb, D. J., Jackson, D. A., Gavrilov, L. R., Hand, J. W., Zhang, L. and Bennion, I., "Infibre Bragg gratings for ultrasonic medical applications," Meas. Sci. Tech. 8, 1050-1054 (1997).

[3] Fisher, N. E., Webb, D. J., Pannell, C. N., Jackson, D. A., Gavrilov, L. R., Hand, J. W., Zhang, L. and Bennion, I., "Ultrasonic hydrophone based on short in-fiber Bragg gratings," App. Opt. 37, 8120-8128 (1998).

[4] Wild, G. and Hinckley, S., "Acousto-ultrasonic optical fiber sensors: Overview and state-of-the-art," IEEE J. Sens 8(7),1184-1193 (2008).

[5] Flax, L., Cole, J. H., De Paula, R. P. and Bucaro, J. A., "Acoustically induced birefringence in optical fibers" J. Opt. Soc. Am. 72(9), 1159-1162 (1982).

[6] K. S. Chiang, H. L. W. Chan, and J. L. Gardner, "Detection of high-frequency ultrasound with a polarizationmaintaining fiber," IEEE J. Lightwave Tech. 8(8), 1221-1227 (1990). 